\title{
ARTICLE
}

\section{Notch regulates BMP responsiveness and lateral branching in vessel networks via SMAD6}

\author{
Kevin P. Mouillesseaux ${ }^{1,2, \star}$, David S. Wiley ${ }^{1, \star}$, Lauren M. Saunders ${ }^{1}$, Lyndsay A. Wylie ${ }^{3}$, Erich J. Kushner ${ }^{1}$, \\ Diana C. Chong ${ }^{3}$, Kathryn M. Citrin ${ }^{1}$, Andrew T. Barber ${ }^{1}$, Youngsook Park ${ }^{4}$, Jun-Dae Kim ${ }^{5}$, Leigh Ann Samsa ${ }^{6}$, \\ Jongmin Kim ${ }^{4}$, Jiandong Liư, 6 , Suk-Won Jin ${ }^{5,8}$ \& Victoria L. Bautch,2,3,7
}

Functional blood vessel growth depends on generation of distinct but coordinated responses from endothelial cells. Bone morphogenetic proteins (BMP), part of the TGF $\beta$ superfamily, bind receptors to induce phosphorylation and nuclear translocation of SMAD transcription factors (R-SMAD1/5/8) and regulate vessel growth. However, SMAD1/5/8 signalling results in both pro- and anti-angiogenic outputs, highlighting a poor understanding of the complexities of BMP signalling in the vasculature. Here we show that BMP6 and BMP2 ligands are pro-angiogenic in vitro and in vivo, and that lateral vessel branching requires threshold levels of R-SMAD phosphorylation. Endothelial cell responsiveness to these pro-angiogenic BMP ligands is regulated by Notch status and Notch sets responsiveness by regulating a cell-intrinsic BMP inhibitor, SMAD6, which affects BMP responses upstream of target gene expression. Thus, we reveal a paradigm for Notch-dependent regulation of angiogenesis: Notch regulates SMAD6 expression to affect BMP responsiveness of endothelial cells and new vessel branch formation.

\footnotetext{
${ }^{1}$ Department of Biology, The University of North Carolina at Chapel Hill, Chapel Hill, North Carolina 27599, USA. ${ }^{2}$ Lineberger Comprehensive Cancer Center, The University of North Carolina at Chapel Hill, Chapel Hill, North Carolina 27599, USA. ${ }^{3}$ Curriculum in Genetics and Molecular Biology, The University of North Carolina at Chapel Hill, Chapel Hill, North Carolina 27599, USA. ${ }^{4}$ Department of Life Systems, Sookmyung Women's University, Seoul, Korea.

${ }^{5}$ Section of Cardiovascular Medicine, Department of Internal Medicine, Yale Cardiovascular Research Center, School of Medicine, Yale University, New Haven, Connecricut 06511, USA. ${ }^{6}$ Department of Pathology and Laboratory Animal Medicine, The University of North Carolina at Chapel Hill, Chapel Hill, North Carolina 27599, USA. ${ }^{7}$ McAllister Heart Institute, The University of North Carolina at Chapel Hill, Chapel Hill, North Carolina 27599 , USA. ${ }^{8}$ School of Life Sciences and Cell Logistics Research Center, Gwangju Institute of Science and Technology, Gwangju, Korea. * These authors contributed equally to this work. Correspondence and requests for materials should be addressed to V.L.B. (email: bautch@med.unc.edu) or to S.-W.J. (email: suk-won.jin@yale.edu).
} 
B lood vessel networks expand via endothelial cell (EC) sprouting, migration, anastomosis and lumenization to form new conduits, a process called sprouting angiogenesis $^{1}$. Heterogeneous yet coordinated responses to pro-angiogenic signals are essential for proper angiogenesis and for subsequent maintenance of a functional vasculature $e^{2-6}$. Numerous signalling pathways integrate to provide this heterogeneity in ways that are not completely understood ${ }^{5,7,8}$.

Bone morphogenetic protein (BMP) signalling is essential to the proper form and function of blood vessels ${ }^{8-14}$. BMPs belong to the tranforming growth factor- $\beta$ superfamily of secreted growth factors and they signal through cell-surface heterotetramers of type II and type I serine/threonine kinase receptors ${ }^{15,16}$. Phosphorylation of type I receptors by type II receptors induces phosphorylation of the receptor-associated SMADs 1, 5 and 8 (R-SMADs 1/5/8). R-SMAD phosphorylation induces association with SMAD4 and this complex translocates to the nucleus, to regulate expression of target genes. SMAD6 is an inhibitory SMAD that competitively binds type I receptors or SMAD4 to inhibit SMAD1/5/8 phosphorylation or nuclear translocation, respectively, and downregulate BMP signalling ${ }^{17,18}$.

Recent studies highlight novel roles for BMPs during angiogenesis $^{8-14}$. Global genetic deletion of SMAD5 in mice led to multiple angiogenesis defects during embryogenesis ${ }^{19}$. EC-selective deletion of SMAD1 and SMAD5 severely impaired angiogenesis, resulting in defective yolk sac and cranial vasculature, and SMAD1/5 double knockout EC displayed reduced proliferation and migration, implicating BMP signalling in cellular processes essential to angiogenesis ${ }^{8}$. In zebrafish, Bmp2b is a venous-specific pro-angiogenic signal that promotes Vegfaa-independent sprouting from the posterior cardinal vein ${ }^{9}$. The type I BMP receptor ALK1 is either anti-angiogenic or pro-angiogenic when inhibited by Fc-conjugation or a highly specific blocking antibody, respectively ${ }^{10,13}$. These data suggest that the angiogenic activity of BMP ligands is context dependent.

EC differentially respond to BMP signalling9 ${ }^{9}$, but how BMP responsiveness is set remains largely unknown. Therefore, we sought to identify a mechanism by which EC intrinsically regulate the magnitude of their responses to BMP ligands. Previous studies indicated that BMP6 and BMP2 can be pro-angiogenic $8,9,20$. Here we show that these ligands are pro-angiogenic, and that lateral vessel branching requires threshold levels of R-SMAD phosphorylation. EC responsiveness to pro-angiogenic BMP ligands is regulated by Notch status. Notch sets responsiveness by regulating the cell-intrinsic BMP inhibitor, SMAD6 and SMAD6 affects BMP responses upstream of target gene expression, revealing a new paradigm for Notch-dependent regulation of angiogenesis.

\section{Results}

BMP2 and BMP6 promote lateral vessel branching. We first determined the effects of BMP6 and BMP2 on vessel branching in a three-dimensional (3D) sprouting angiogenesis assay ${ }^{21}$ using human umbilical vein endothelial cells (HUVEC). Addition of exogenous BMP6 or BMP2 significantly increased both branching frequency and branch angle compared with controls (Fig. 1a-d and Supplementary Fig. 1a-d). Exogenous BMP2 had a similar effect on branching frequency in mouse embryonic stem (ES) cell-derived vessels (Supplementary Fig. 1e-g). Consistent with a more highly branched phenotype, both ligands increased the percentage of nuclei in the tip position of growing HUVEC sprouts (Fig. 1e-g and Supplementary Fig. 1h-j). Moreover, the BMP-induced branching phenotype was remarkably well- organized, in contrast to the effects of elevated vascular endothelial growth factor (VEGF)-A signalling, which often lead to gross dysmorphogenesis ${ }^{22,23}$. Thus, BMP6 and BMP2 are pro-angiogenic and increase vascular density by inducing new branch formation. Furthermore, the increased branch angles lead to lateralization of the branching pattern and suggest that pro-angiogenic BMP signalling tunes vessel branching between a 'bush-like' and a 'bamboo-like' vessel pattern, in a liganddependent manner.

We hypothesized that BMP-induced branching depended on EC sprouting in response to activation and nuclear localization of phosphorylated SMADs 1 and 5 (pSMAD1/5). We examined levels of nuclear pSMAD1/5 in EC during sprouting angiogenesis and found a striking inverse correlation between the distance of nuclei from the sprout tip and intensity of nuclear pSMAD1/5 staining (Fig. 1h,j,m). Thus, EC with the highest levels of BMP signalling were more likely to be in the tip cell position. As expected, short-term BMP6 treatment significantly increased overall nuclear pSMAD1/5 levels compared with controls (Fig. 1i-k and Supplementary Fig. 1k). However, although acute BMP6 exposure significantly increased the average nuclear pSMAD1/5 levels in stalk cells, it had only a modest and nonsignificant effect in tip cells (Fig. 11), suggesting that EC at sprout tips have maximal BMP pathway activation. In fact, stalk EC in BMP6-treated sprouts had average nuclear pSMAD1/5 levels comparable to levels in tip EC of controls (Fig. 11), suggesting that BMP signalling increases vessel branching by inducing additional tip cells from the pool of stalk cells. This idea is also consistent with the finding that stalk cells, even after BMP stimulation, retain extensive heterogeneity in nuclear pSMAD1/5 levels and their pSMAD1/5 levels do not as strongly correlate with position in the sprout as controls (Fig. 11,m).

To verify the necessity of SMAD $1 / 5$ for lateral branch formation, we reduced the levels of each protein via small interference RNAs (siRNAs) and analysed effects on sprouting. Reduction of either SMAD1 or SMAD5 (Supplementary Figs 11,m and $5 \mathrm{~b}$ ) reduced BMP6-induced lateral branching. Although SMAD1/5 signalling has also been implicated in stalk cell maintenance ${ }^{5}$, this effect is thought to be downstream of BMP9 engagement with complexes that include the type I receptor ALK1 and induce vascular quiescence ${ }^{13,24,25}$. To determine whether ALK1 signalling contributes to the effects we observed, we reduced ALK1 levels via siRNA and found no effect on BMP6-dependent increases in lateral branching (Supplementary Figs 1n and 5b), suggesting that BMP6-induced branching occurs independently of BMP9/ALK1 signalling. Thus, pSMAD1/5 is required to mediate the pro-angiogenic effects of BMP6 in EC independent of effects from ALK1 signalling. Taken together, these data suggest that BMP-dependent lateral vessel branching depends on EC that are 'tunable' and have an innate set point of BMP responsiveness. For each EC, this innate responsiveness sets a ligand threshold required to produce sufficient pro-angiogenic pSMAD1/5 signalling and provoke an EC response. In this scenario, short-term BMP6 treatment increases the number of EC that achieve the threshold and are capable of responding, leading to additional lateral branching.

Notch sets EC BMP responsiveness. If BMP responsiveness is innate to EC, then factors that set this responsiveness and the extent of R-SMAD phosphorylation are predicted to profoundly affect BMP-dependent vascular architecture. Notch signals via its intracellular domain (NICD) and this signalling represses the tip cell phenotype and supports a non-branching stalk cell phenotype ${ }^{26-29}$. Although recent work describes Notch-BMP pathway crosstalk at the level of target gene expression ${ }^{8,13,30,31}$, it is not clear whether Notch signalling alters the responsiveness of EC to BMP via effects on upstream BMP pathway components. 

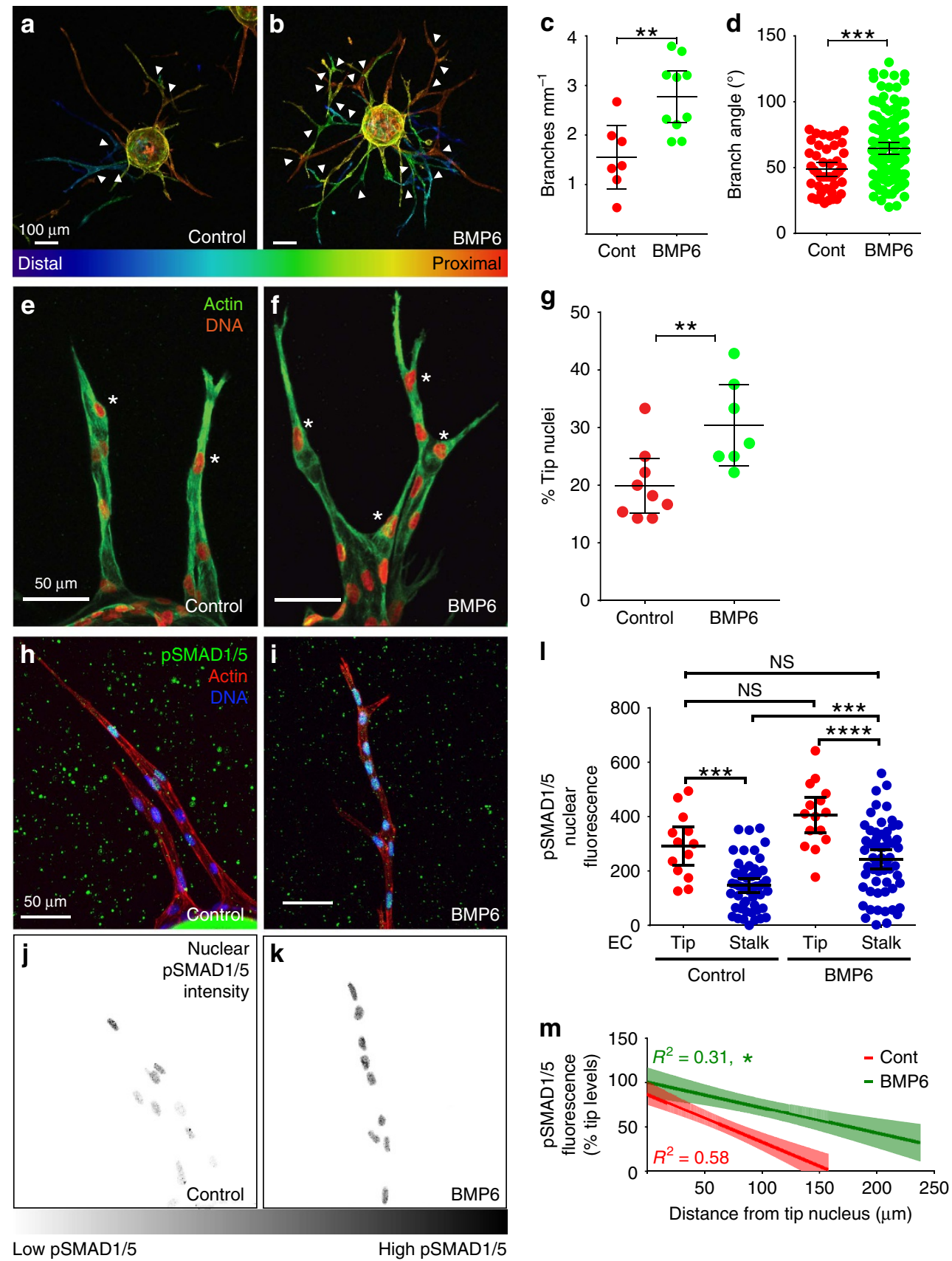

Figure 1 | BMP signalling promotes lateral branching of angiogenic sprouts. (a,b) HUVEC 3D sprouting assay with BMP6, representative of three independent experiments, stained with phalloidin (actin) and depth encoded. (c,d) Quantification of (c) branches per mm ( $n=7$ control and 10 BMP6 beads) and (d) branch angle ( $n=43$ control and 133 BMP6 angles). Error bars, mean $\pm 95 \%$ confidence interval $(\mathrm{Cl})$. ${ }^{\star \star} P \leq 0.01$; ${ }^{\star \star \star} P \leq 0.001$ by Student's t-test. (e,f) Sprouting HUVEC visualized with phalloidin (actin) and DRAQ7 (DNA). (g) Tip nuclei/total nuclei per field, representative of three independent experiments ( $n=9$ control and 7 BMP6 sprouts). Error bars, mean $\pm 95 \% \mathrm{Cl}$. ${ }^{\star \star} P \leq 0.01$ by Student's $t$-test. (h-k) HUVEC $3 D$ sprouting assay $6 \mathrm{~h}$ post-BMP6 treatment, visualized for nuclear pSMAD1/5, phalloidin (actin) and DRAQ7 (DNA). Images are three-channel compressed z-stacks (h,i) or single-channel compressed $z$-stack heat maps of pSMAD1/5 staining intensity (j,k). Scale bar, $50 \mathrm{~mm}$. (I) Quantification of mean pSMAD1/5 fluorescence intensity per nucleus, representative of 2 independent experiments $(n=13$ control tip, 50 control stalk, 15 BMP6 tip, and 58 BMP6 stalk EC). Error bars, mean $\pm 95 \% \mathrm{Cl}$. NS, not significant; ${ }^{\star \star \star} P \leq 0.001$ and ${ }^{\star \star \star \star} P \leq 0.0001$ by one-way analysis of variance with Tukey's post-hoc test. (m) Best-fit correlation (solid line) with $95 \% \mathrm{Cl}$ intervals (filled areas) of indicated parameters. ${ }^{\star} P \leq 0.05$ by linear regression.

In the developing zebrafish, venous EC are BMP sensitive and form ectopic sprouts after heat-shock induction of $\mathrm{Bmp} 2 \mathrm{~b}$, whereas intersegmental vessels (ISVs) are relatively unresponsive at $24-48 \mathrm{hpf}$ (ref. 9). To investigate regulators of BMP responsiveness in this model, we used $T g(T p 1 b g l o b: e G F P)$ (Notch reporter) zebrafish embryos and asked how Notch activity and BMP sensitivity align in the vasculature. Notch reporter activity was strong in the dorsal aorta (DA) and ISVs, consistent with other reports 32,33 , and these vessels do not respond to ectopic BMP ligand ${ }^{9}$; however, the reporter signal was undetectable in the BMP-responsive caudal vein plexus (CVP) (Fig. 2a,b). To determine whether Notch influences BMP responsiveness, we induced Notch signalling via heat-shock induction of NICD. As we have described, ectopic induction of 

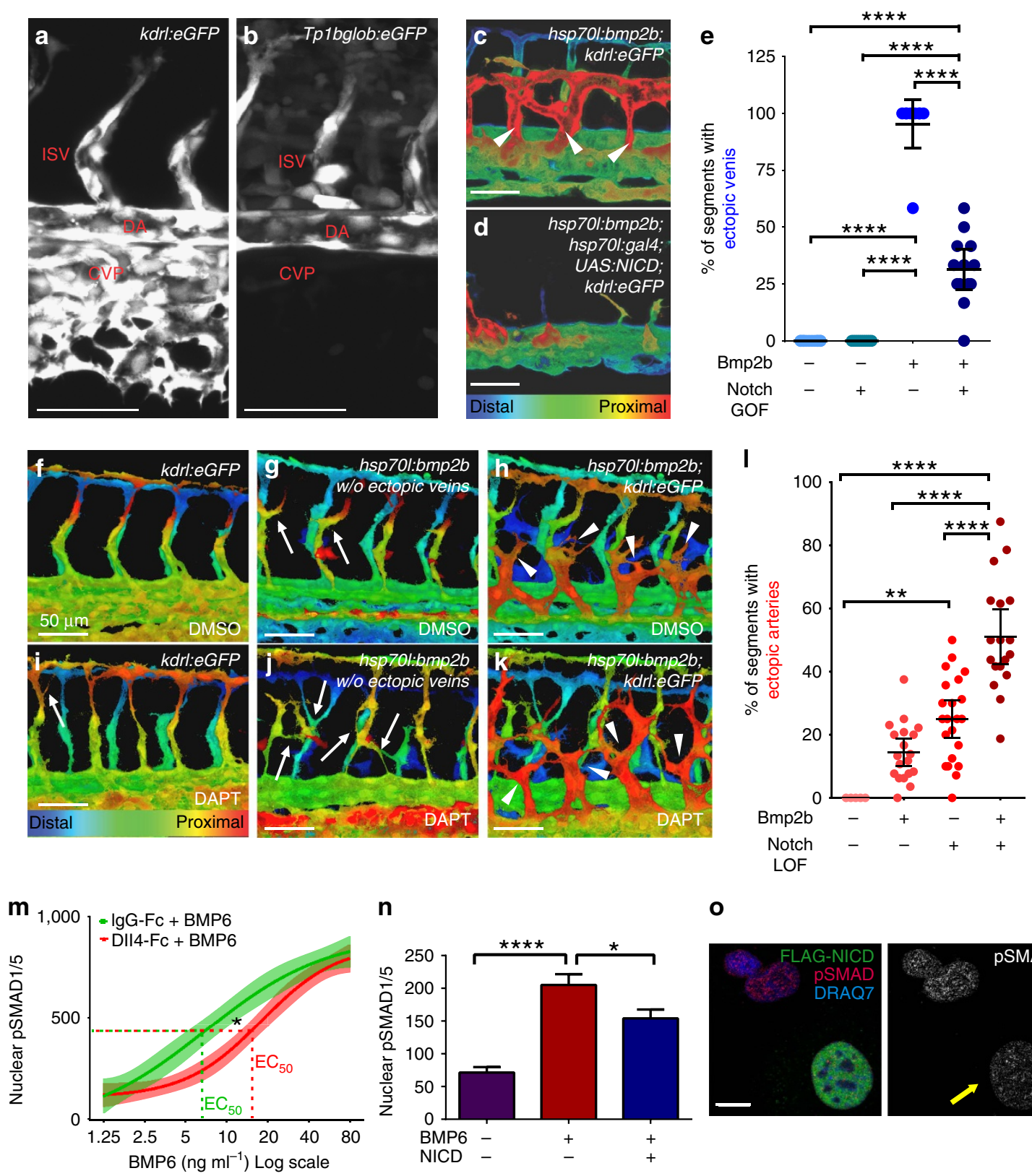

n

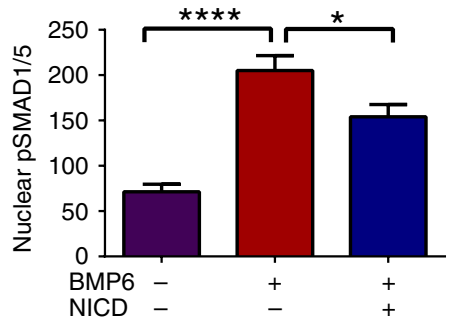

o

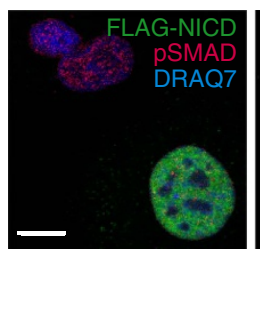

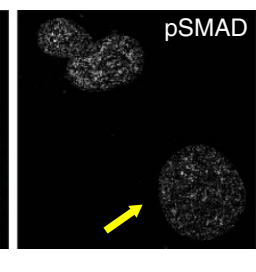

Figure 2 | Notch regulates BMP responsiveness. (a,b) Lateral view of vessel-specific (a) or Notch-activated (b) GFP expression in 28 hpf fish embryos. Scale bar, $50 \mu \mathrm{m}$. (c,d) Depth-encoded lateral view of $44 \mathrm{hpf}$ fish embryos heat-shocked at $26 \mathrm{hpf}$. Scale bar, $50 \mu \mathrm{m}$. (c) Tg(hsp70l:bmp2b) embryos overexpress Bmp2b; (d) Tg(hsp70l:bmp2b); Tg(UAS:NICD); Tg(hsp70l:gal4) overexpress both Bmp2b and NICD. (e) Quantification of ectopic venous sprouts, representative of two independent experiments. Data points, individual embryos ( $n=12 \mathrm{WT}, 11 \mathrm{Tg}$ (hsp70l:Gal4); $\mathrm{Tg}(\mathrm{UAS}: \mathrm{NICD}), 9 \mathrm{Tg}(\mathrm{hsp} 70 \mathrm{l}: \mathrm{bmp} 2 b)$, 13 compound transgenics). Error bars, mean $\pm 95 \% \mathrm{Cl}$. ${ }^{\star \star \star \star} P \leq 0.0001$ by one-way analysis of variance (ANOVA), with Tukey's post-hoc test. (f-k) Depth-encoded compressed z-stack lateral views of $44 \mathrm{hpf}$ embryos heat shocked at $26 \mathrm{hpf}$ and treated with vehicle (f-h) or DAPT (i-k) from $10 \mathrm{hpf}$, representative of three independent experiments. (f,i) WT embryos; (g,j) Tg(hsp70l:bmp2b) embryos with ectopic venous $z$-slices removed to visualize intersegmental arteries; (h,k) $T g(h s p 70 l: b m p 2 b)$ embryos. (I) quantification of ectopic arterial sprouts. Data points, individual embryos $(n=27$ WT/dimethyl sulfoxide (DMSO), $26 \mathrm{Bmp} 2 \mathrm{~b} / \mathrm{DMSO}, 23 \mathrm{WT} / \mathrm{DAPT}$ and $26 \mathrm{Bmp} 2 \mathrm{~b} / \mathrm{DAPT}$ ). Error bars, mean $\pm 95 \% \mathrm{Cl} .{ }^{\star} P \leq 0.05,{ }^{\star \star} P \leq 0.01 \mathrm{and}$ ${ }^{\star \star \star \star} P \leq 0.0001$ by Kruskal-Wallis with Dunn's post-hoc test. DA, dorsal aorta; DV, dorsal vein; ISV, intersegmental vessel; VV, ventral vein. (m) BMP6 twofold dose-response curve (indicated on $x$ axis) in HUVEC after Notch activation (DII4-Fc, red line) versus control (IgG-Fc, green line), representative of two independent experiments. Data are four-parameter best-fit curves (solid lines) $\pm 95 \%$ confidence bands (filled areas). ${ }^{\star} P \leq 0.05$ by nonlinear regression. Quantification (n) and panels (o) of nuclear PSMAD1/5 expression in individual HUVEC with indicated conditions, representative of two independent experiments. Yellow arrow, EC expressing FLAG-NICD. Scale bar, $10 \mu \mathrm{m}$. Error bars, mean \pm s.e.m. ${ }^{\star} P \leq 0.05$ and ${ }^{\star \star \star \star} P=0.0001$ by one-way ANOVA with Tukey's post-hoc test.

Bmp2b led to excessive sprouting from the CVP (Fig. 2c,e) ${ }^{9}$. However, concomitant induction of Bmp2b and NICD significantly reduced the frequency of CVP sprouts, suggesting that ectopic Notch signalling dampens the sensitivity of EC to
BMPs (Fig. 2d,e). Conversely, to determine whether arterial EC could be sensitized to Bmp2b overexpression, we blocked Notch signalling by treatment with $\mathrm{N}$-[2S-(3,5-difluorophenyl)acetyl]-Lalanyl-2-phenyl-1,1-dimethylethyl ester-glycine (DAPT), a 
$\gamma$-secretase inhibitor that prevents cleavage and release of NICD. Bmp2b induction induced a low level of ectopic vessels from arterial EC (Fig. 2f-h,l) and DAPT treatment alone induced some ectopic arterial angiogenesis, consistent with previous reports (Fig. 2i,l) ${ }^{5,27}$. However, Notch inhibition combined with Bmp2b induction resulted in a significantly higher frequency of ectopic arteries compared with either manipulation alone (Fig. 2j-1). These results indicate that Notch is an intrinsic regulator of the magnitude of the BMP response in EC in vivo.

To quantitatively determine the impact of Notch manipulations on BMP pathway activation, we determined nuclear pSMAD1/5 levels on exposure of HUVEC to different amounts of ligand. A twofold serial dose-response curve to BMP6 yielded a prototypical sigmoidal semi-log curve for BMP-mediated EC activation, as measured by nuclear pSMAD1/5 levels (Supplementary Fig. 2a,b). We next tested the effect of Notch activation by plating HUVEC onto Fc-conjugated Dll4 ligand (Dll4-Fc) before short-term treatment with BMP6 and found that the $\mathrm{EC}_{50}$ for $\mathrm{BMP}$-mediated $\mathrm{EC}$ activation increased significantly compared with controls (Fig. 2m). We confirmed, using inducible NICD expression in HUVEC, that elevated Notch signalling increased the $\mathrm{EC}_{50}$ (Supplementary Fig. 2c). This relationship also held at the single-cell level, as EC expressing NICD had reduced levels of pSMAD1/5 (Fig. 2n,o). Conversely, HUVEC treated with siRNA targeting Notch1 (Supplementary Figs 2d and 5c) were more sensitive to lower concentrations of BMP6 relative to controls (Supplementary Fig. 2e) and they exhibited increased branching with equivalent BMP6 stimulation (Supplementary Fig. 2f-j). The results of in vivo and in vitro Notch manipulations support our hypothesis that Notch regulates the innate BMP responsiveness of $\mathrm{EC}$, and that the increased BMP responsiveness of EC with low Notch signalling promotes lateral branching.

SMAD6 integrates Notch and pro-angiogenic BMP responsiveness. Notch regulates VEGF signalling by modulating levels of VEGF receptor $\mathrm{RNAs}^{34,35}$. Therefore, we reasoned that Notch would regulate BMP responsiveness via expression of BMP receptors. Surprisingly, we detected no significant changes in expression levels of several type I and type II BMP receptors after Notch stimulation of HUVEC via Dll4-Fc plating or Notch blockade via DAPT (Supplementary Fig. 3a,b). BMP signalling is also intrinsically regulated by an intracellular inhibitory protein, SMAD6 (refs 17,18,36,37) and SMAD6 messenger RNA levels increased with Notch stimulation and decreased with Notch blockade in HUVEC (Fig. 3a). This relationship was maintained at the single-cell level, as EC expressing NICD had elevated levels of SMAD6 protein (Fig. 3b,c). In other cell types, SMAD6 inhibits BMP signalling by preventing R-SMAD phosphorylation and nuclear localization ${ }^{17,18}$, but its activity in EC and effects on angiogenesis are unknown. Therefore, we generated HUVEC expressing doxycycline-inducible SMAD6 fused to tdTomato. The tagged SMAD6 protein reacted with a SMAD6 antibody by immunofluorescence (Supplementary Fig. 3c-f) and suppressed nuclear pSMAD1/5 levels in HUVEC in a cell autonomous and dose-dependent manner (Fig. 3d,e). Conversely, reduction of SMAD6 protein levels via siRNA knockdown (Supplementary Figs $3 \mathrm{~g}$ and $5 \mathrm{~d}$ ) increased BMP6-induced nuclear pSMAD1/5 (Fig. 3f). These findings show that an intrinsic BMP pathway inhibitor, SMAD6, modulates BMP signalling in EC. As elevated pSMAD levels were associated with increased lateral branching and SMAD6 suppressed BMP signalling, we hypothesized that loss of SMAD6 would increase BMP responsiveness of EC and promote branching. Consistent with this hypothesis, reduced SMAD6 levels via siRNA significantly increased branching of sprouting HUVEC with added exogenous BMP6 (Fig. 3g-k). These results show that SMAD6 regulates angiogenesis, probably by intrinsically modulating the magnitude of $\mathrm{EC}$ responses to BMP inputs.

To determine the functional relevance of SMAD6 in vivo, we first examined expression by fluorescence-activated cell sorting (FACS) in EC from zebrafish embryos that expressed the Notch signalling reporter (Fig. 2a). Zebrafish have two SMAD6 orthologues, smad6a and smad6b. We assessed expression of smad6b in EC in vivo, because its sequence is more homologous to that of human SMAD6. EC sorted for high levels of Notch reporter signalling had significantly elevated levels of RNAs that are expected to be elevated in arterial Notch-positive EC such as notch $1 b$ and ephrinb2. They also had significantly elevated levels of smad6b RNA relative to EC sorted from the same embryos with little or no Notch reporter signalling (Fig. 4a). We next manipulated embryonic SMAD6 expression. As global manipulations of smad $6 \mathrm{~b}$ are predicted to profoundly perturb dorsal-ventral axis formation in zebrafish embryos ${ }^{38}$, we used the Tol2 system to generate F0 mosaic embryos expressing green fluorescent protein (GFP)-tagged smad6b, or GFP alone, under control of the vascular-specific fli1 promoter. We predicted that Smad6b expression would preferentially affect BMP-responsive EC with low Notch levels, such as those in the cardinal vein and the CVP. EC expressing Smad6b-eGFP were significantly reduced in the cardinal vein and CVP, and enriched in the DA and ISVs, compared with EC expressing only GFP (Fig. 4b-d), consistent with our finding that Smad6b expression is elevated in EC of embryos with high Notch signalling reporter expression, presumably including EC from the DA and ISVs. These findings suggest that forced Smad6b expression prevents EC from colonizing the vein and CVP where Notch signalling is low, but is irrelevant in arterial EC where Notch signalling is relatively high and support a role for SMAD6 in angiogenesis in vivo.

We next asked whether reduced levels of smad6b affect blood vessel branching and predicted that if SMAD6 is important for Notch-mediated effects in vivo, its reduction would more profoundly affect Notch-dependent ISV sprouting that is normally refractory to BMP ${ }^{9}$. To overcome the effects of global smad6b manipulations on dorsal-ventral axis formation, we developed a tissue-specific mRNA knockdown system for zebrafish based on a CRISPRi strategy $y^{39-42}$. However, CRISPRi is poorly efficient in eukaryotic cells ${ }^{40}$; thus, dCas9 was fused to the Drosophila engrailed repressor domain ${ }^{43}$ to generate dCas9Engrailed Repressor domain (EnR), a chimeric transcriptional repressor protein that is targeted by short guide (sg) RNAs. We validated the efficacy of dCas9-EnR in global knockdown using sgRNAs targeting bmp6 or smad6b and observed the expected dorsalization (bmp6 loss-of-function) or ventralization (smad6b loss-of-function) phenotypes (Supplementary Fig. 4a-d). We also validated sgRNA effects at the mRNA level by quantitative reverse transcriptase-PCR (qRT-PCR) for the targeted genes (Supplementary Fig. 4e). Next, transgenic embryos expressing a fli1 promoter-driven $d$ Cas9-EnR were generated using the Tol2 system. F1 embryos expressing vascular restricted dCas9-EnR were indistinguishable from wild-type (WT) siblings and dCas9-EnR mRNA expression was confirmed by RT-PCR (Supplementary Figs $4 \mathrm{f}-\mathrm{h}$ anf $5 \mathrm{e}$ ). To determine whether Smad6b regulates BMP responsiveness in vivo, we injected sgRNAs targeting smad6b into $\mathrm{Tg}(f l i 1: d C a s 9-E n R) ; \operatorname{Tg}($ hsp701: $b m p 2 b) ; T g(k d r l: G F P)$ embryos, then heat shocked to induce ectopic Bmp2b expression at $26 \mathrm{hpf}$, well beyond the early developmental window leading to axis defects. WT heat-shocked embryos without sgRNAs had normal ISV formation (Supplementary Fig. 4i,j,m). Likewise, $T g(f l i: d C a s 9-E n R) ; T g(k d r l: G F P)$ embryos injected with scrambled sgRNAs or smad6b sgRNAs and heat-shocked had normal ISV formation (Fig. 4e,f,i). Heat-shock induction of Bmp $2 \mathrm{~b}$ expression did not perturb axis formation, 

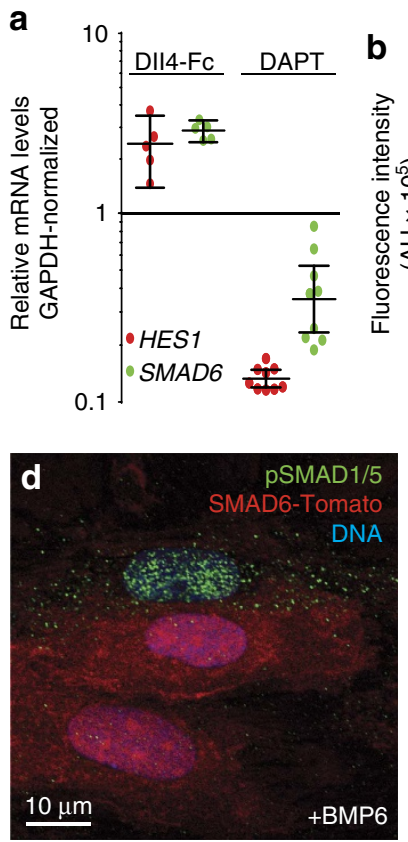

b

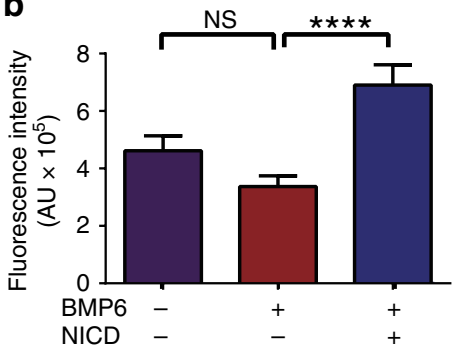

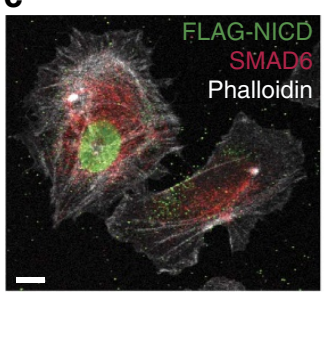

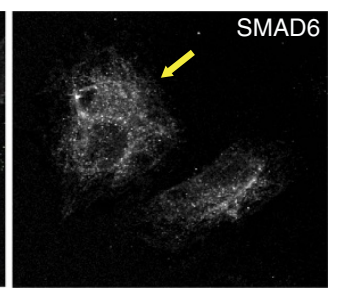

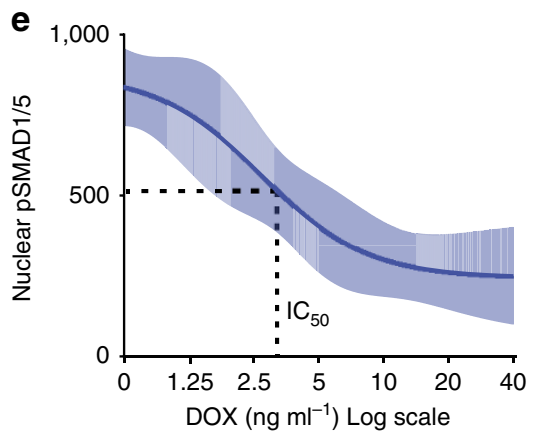
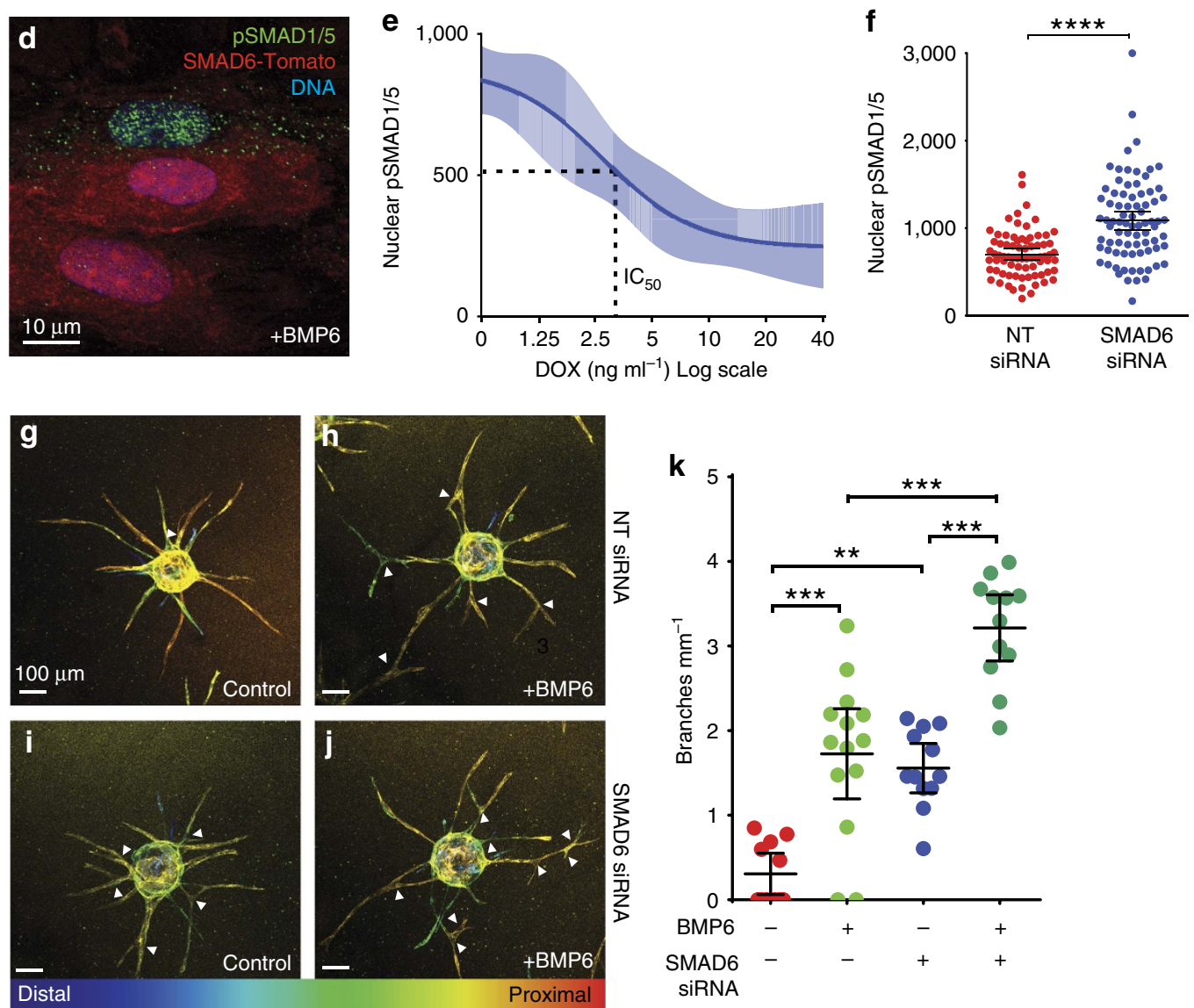

Figure 3 | SMAD6 is Notch regulated and anti-angiogenic. (a) qRT-PCR for HES1 or SMAD6 with Notch gain-of-function (DII4-Fc) or loss-of-function

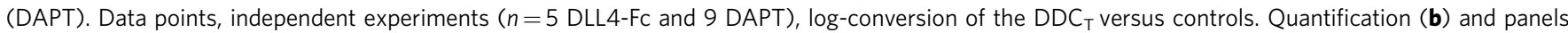
(c) of nuclear SMAD6 expression in individual HUVEC with indicated parameters, representative of two independent experiments. Scale bar, $10 \mu \mathrm{m}$. Yellow arrow, EC expressing FLAG-NICD. Error bars, mean \pm s.e.m., ${ }^{\star \star \star \star} P<0.0001$ by one-way analysis of variance using Tukey's post-hoc; NS, not significant. (d) Stable lentivirally transduced HUVEC infected with SMAD6-tdTomato conditonally expressing virus, stimulated with doxycycline (DOX) and stained as indicated. (e) Quantification of twofold dose-response (indicated on $x$ axis) to DOX (SMAD6-tdTomato expression) after 90 min BMP6 treatment, representative of two independent experiments. Readout is nuclear pSMAD1/5. Data are four-parameter best-fit curves (solid lines) $\pm 95 \%$ confidence bands (filled areas). (f) HUVEC transfected with indicated siRNAs, then treated with $40 \mathrm{ng} \mathrm{ml}^{-1}$ BMP6 for 90 min before fixation and staining for nuclear pSMAD1/5, representative of two independent experiments ( $n=75 \mathrm{NT}$ and 80 SMAD6 siRNA HUVEC). Error bars, mean $\pm 95 \% \mathrm{Cl}$. ${ }^{\star \star \star \star} P \leq 0.0001$ by Student's $t$-test. ( $\mathbf{g}$-j) HUVEC 3D sprouting assay with indicated siRNAs and treatment, visualized with phalloidin (actin) and depth-encoded in a compressed z-stack, representative of three independent experiments. (k) Quantification of branching ( $n=11$ NT/control, 13 NT/BMP6, 12 SMAD6 siRNA/control and 11 SMAD6 siRNA/BMP6 beads). Data points, individual beads; bars, mean $\pm 95 \% \mathrm{Cl}$. ${ }^{\star \star} P \leq 0.01$ and ${ }^{\star \star \star} P \leq 0.001$ by Kruskal-Wallis with Dunn's post-hoc test.

but induced some ectopic ISV formation in controls and $\operatorname{Tg}(f l i: d C a s 9-E n R) ; \quad T g(h s p 701: b m p 2 b) ; T g(k d r l: G F P) \quad$ embryos injected with scrambled sgRNAs (Fig. 4g,i and Supplementary Fig. $4 \mathrm{k}, \mathrm{m}$ ). However, the frequency of ectopic ISV formation significantly increased in transgenic embryos overexpressing Bmp2b and injected with smad6b sgRNAs (Fig. 4h,i; Supplementary Fig. $41, \mathrm{~m}$ ). Remarkably, the phenotype of BMP-overexpressing, vascular smad6b knockdown embryos resembles that of BMPoverexpressing embryos with reduced Notch signalling. Combined, these in vivo results support a model whereby SMAD6 represses BMP responsiveness and vessel branching.

Based on the similarity of phenotypes in vivo and the Notch responsiveness of SMAD6 RNA and protein in EC, we further explored the mechanism by which Notch regulates SMAD6 gene 
expression. Analysis of the SMAD6 5'-promoter region revealed a consensus sequence for Recombination Signal Binding Protein for Immunoglobulin Kappa J Region (RBPJ) binding to DNA, which is required for Notch-dependent transcription in cells. A chromatin immunoprecipitation (ChIP) assay using two separate antibodies to RBPJ revealed that RBPJ binds at the identified site in the SMAD6 promoter (Fig. 5a and Supplementary Fig. 5a), suggesting that Notch directly regulates transcription of SMAD6 RNA.
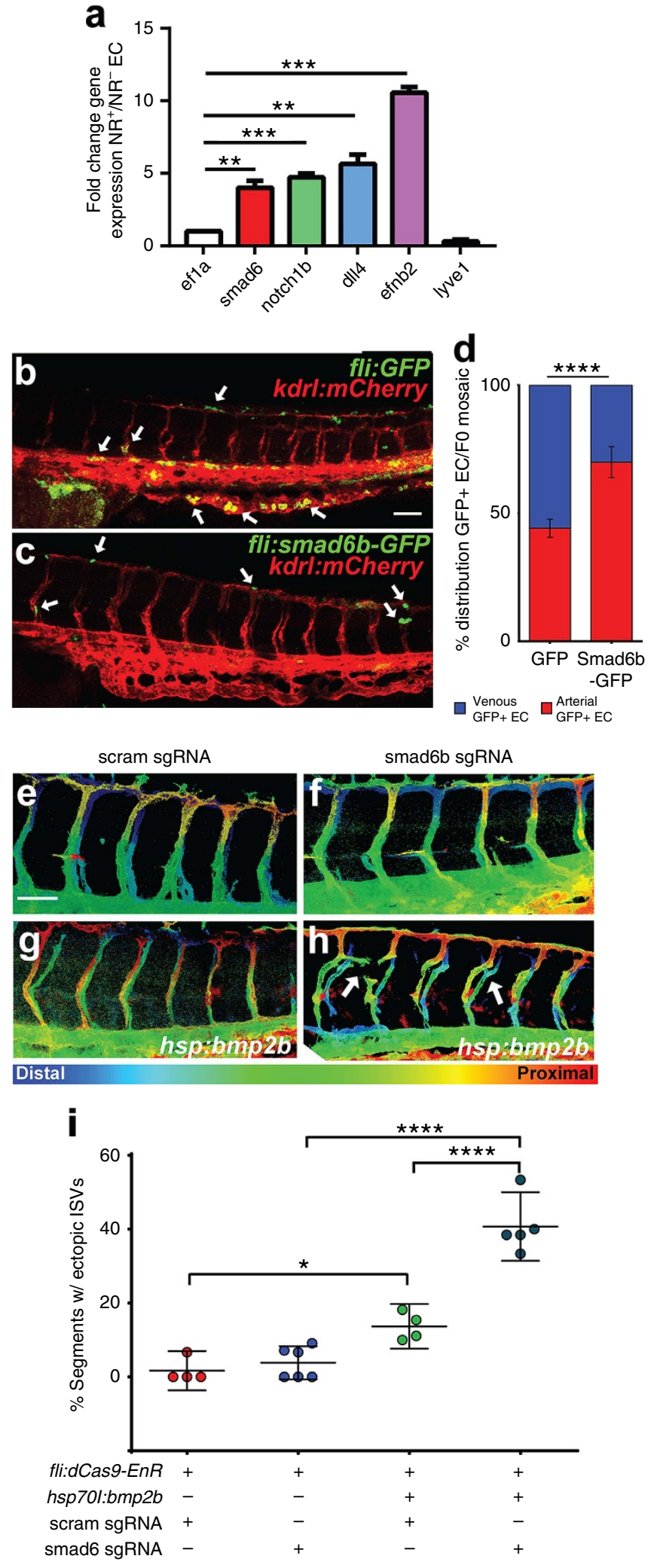

Finally, we hypothesized that SMAD6 is functionally downstream of Notch in regulating the BMP responsiveness of EC. We tested this hypothesis by activating Notch in HUVEC with reduced levels of SMAD6, and we assessed BMP pathway signalling via nuclear localization of pSMAD1/5. Short-term BMP6 treatment of HUVEC led to elevated nuclear pSMAD levels that were significantly suppressed by Notch stimulation (Fig. 5b,c,f,g,j). In contrast, HUVEC with reduced SMAD6 levels and BMP6 stimulation did not respond to Notch stimulation with suppression of SMAD1/5 activation, but had nuclear pSMAD1/5 levels equivalent to that of HUVEC without Notch stimulation (Fig. 5d,e,h-j). These data indicate that SMAD6 is functionally downstream of Notch and is required to mediate the effects of Notch on BMP responsiveness in EC.

\section{Discussion}

Collectively, our data support a model whereby pro-angiogenic BMPs increase vascular complexity through regulation of lateral branching. The magnitude of intrinsic BMP responsiveness in EC is modulated by Notch-mediated regulation of the BMP inhibitor SMAD6 to 'tune' BMP responsiveness (Fig. 6), and combined effects on branch frequency and angle lead to a more arborized or 'bush-like' vascular network as BMP ligand levels increase. In this model, Notch signalling sets levels of SMAD6 in a given EC and SMAD6 levels regulate the amount of pSMAD1/5 that translocates to the nucleus with a given input of BMP ligand. As ligand levels increase, more EC reach a threshold that allows them to assume a tip cell phenotype and sprout to form a new branch. This includes a subset of EC in a classic 'stalk' position, and our finding of differential BMP responsiveness in the stalk cell compartment indicates dissimilarity among stalk cells. Notch is a major regulator of the tip cell versus stalk cell phenotype in EC of angiogenic sprouts ${ }^{26,44}$, and we now propose that Notch also differentiates stalk cells by modulating expression of SMAD6. We predict that Notch-dependent changes in SMAD6 expression among dynamically competing sprouting EC shifts BMP responsiveness relative to a threshold necessary for lateral branching. Subsequently, as arteries reach homeostasis and Notch signalling becomes more uniform, these changes may lead to more uniform SMAD6 levels and overall dampened BMP responsiveness in arteries versus veins.

Figure 4 | SMAD6 regulates BMP-dependent angiogenesis in vivo.

(a) Quantitative RT-PCR for indicated genes, fold-change Notch reporter $+(N R+) /$ Notch reporter- $(N R-) E C$, relative to elfa. Error bars, mean \pm s.e.m., $N=5$ replicates; one-sample Student's $T$-test, ${ }^{\star \star} P \leq 0.01$ and ${ }^{\star \star \star} P \leq 0.001$. (b-d) FO mosaic transgenic zebrafish embryos (44 hpf) expressing GFP control (b) or GFP-tagged smad6b (c) from the vessel-specific fli promoter in the $T g(k d r l: m$ Cherry) line. Arrows, GFP + EC. (d) Arterial versus venous distribution of GFP + EC quantified as percentage of total GFP + EC on a per-embryo basis. Data bars, average per cent arterial and venous GFP $+\mathrm{EC}, \pm 95 \% \mathrm{Cl}$, representative of three independent experiments ( $n=18$ fli:GFP and 30 fli:smad6b-GFP FO embryos). ${ }^{\star \star \star \star} P \leq 0.0001$ by $\chi^{2}$ analysis (1 degree of freedom). (e-h) Heat-shocked F1 embryos (heat shock at $26 \mathrm{hpf}$, analysed at 44-46 hpf) from Tg(fli:dCas9-EnR) and Tg(hsp70l:bmp2b;Tg(kdrl:GFP) crosses, injected with scrambled (scram) or with smad6b sgRNAs. Arrows, ectopic ISV sprouts. (g,h) have Z-planes with ectopic venous sprouts removed. (i) Quantification of arterial vascular defects (\% segments with ectopic ISVs) in heat-shocked embryos of indicated genotypes, representative of two independent experiments (scram, $n=4$; smad6, $n=6$; scram/hsbmp, $n=4$; smad6/hsbmp, $n=5$ ). Error bars, mean \pm s.e.m.; ${ }^{\star} P \leq 0.05$ and ${ }^{\star * \star \star} P \leq 0.0001$ by one-way analysis of variance, with Tukey's post-hoc test. 

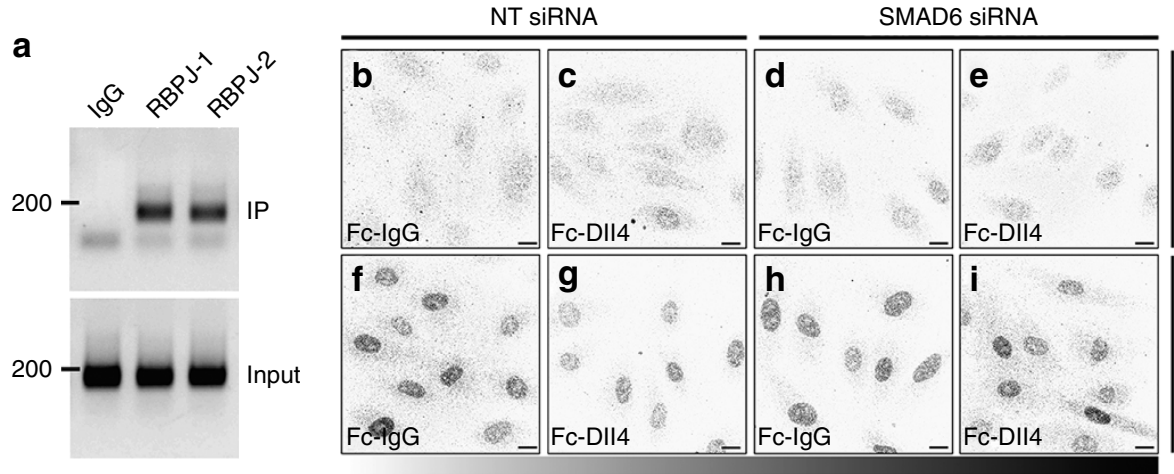

Low pSMAD $1 / 5$

High pSMAD1/5

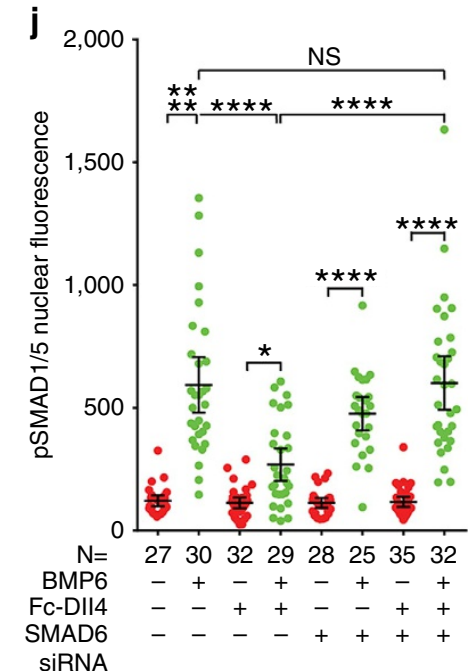

Figure 5 | SMAD6 mediates Notch-dependent suppression of BMP signalling. (a) ChIP assay on micrococcal nuclease-digested HUVEC DNA immunoprecipitated with indicated antibodies and amplified unique primers targeting a single putative RBPJ consensus sequence in the SMAD6 promoter. Left, size marker (bp). (b-i) HUVEC transfected with non-targeting $(\mathbf{b}, \mathbf{c}, \mathbf{f}, \mathbf{g})$ or SMAD6 siRNA (d,e,h,i), plated onto IgG (b,d,f,h) or DII4-Fc (c,e,g,i), then treated with control (b-e) or $50 \mathrm{ng} \mathrm{ml}^{-1}$ BMP6 (f-i) for 90 min before staining for nuclear pSMAD1/5. Scale bar, $10 \mu \mathrm{m}$. (j) Quantification of nuclear pSMAD1/5 fluorescence intensity, representative of three independent experiments. Data points, individual nuclei ( $\mathrm{n}$ indicated on graph); bars, mean $\pm 95 \% \mathrm{Cl} .{ }^{\star} P \leq 0.05,{ }^{\star \star} P \leq 0.01$ and ${ }^{\star \star \star \star} P \leq 0.0001$ by Kruskal-Wallis with Dunn's post-hoc test.

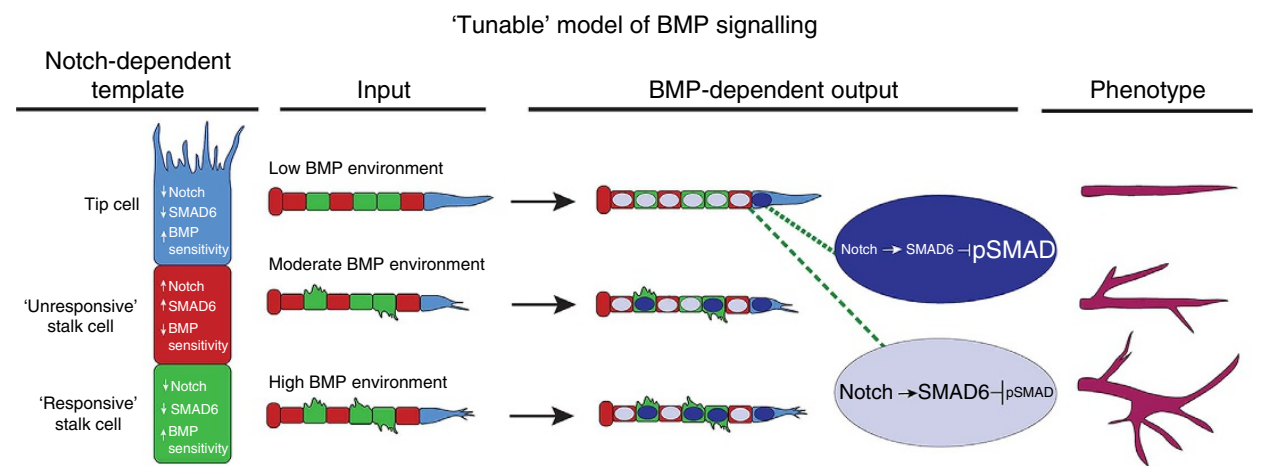

Figure 6 | Model of SMAD6-mediated BMP responsiveness in vessels. The data support a model whereby Notch sets BMP responsiveness of sprouting EC through the intrinsic BMP inhibitor SMAD6, leading to a 'tunable' system that responds to increased BMP ligands with more lateral branching.

Allthough convergence of Notch and BMP pathways at downstream target genes has been described $8,13,30$, our data strongly suggest that intrinsic responsiveness to BMP signals is independently preset by Notch to regulate lateral branching. Recent work suggests that Notch also maintains the stalk cell phenotype via downregulation of Neuropilin-1 (ref. 45). Low Neuropilin-1 in stalk cells relieves inhibition of ALK1 and ALK5, and promotes tranforming growth factor- $\beta$-mediated pSMAD2/3 signalling to maintain the stalk cell phenotype. Our work shows that SMAD6, a Notch-regulated cell-intrinsic BMP pathway inhibitor, modulates EC responses to pro-angiogenic BMP ligands that stimulate pSMAD1/5 signalling and a tip cell phenotype. Thus, Notch-mediated SMAD6 regulation 'tunes' branching responses to BMP ligands among stalk cells and coordinately regulates the magnitude of BMP pathway activation and sprouting, to determine the patterning of growing vessel networks.

\section{Methods}

Cell maintenance and processing. No cell lines used in this study are found in the International cell line authentication committee (ICLAC) commonly misidentified cell line database.
Mouse ES cells were maintained and differentiated as previously described ${ }^{46}$. Briefly, cells were maintained undifferentiated by culture in DMEM media supplemented with conditioned media from 5,637 human bladder cancer cells (ATCC HTB9) and passaged at 2-4 days. For differentiation, ES cells aged for 2-4 days beyond passage were trypsinized, resuspended and inoculated into droplets on a tissue culture plate lid. The lid was inverted, hanging drops cultured for 2 days to generate embryoid bodies (EBs), then inverted again and flushed with media. After culture in a new plate for 1 day, EBs were seeded at 20-30 EBs per well of a 24-well plate and differentiated in DMEM, Hi-glucose, 20\% FCS, $150 \mu \mathrm{M}$ monothioglycerol and $50 \mu \mathrm{g} \mathrm{ml}^{-1}$ gentamicin. Media was changed every 2 days until fixation. Human BMP2 (R\&D Systems 355-BEC) was added at d6 and d7 at $200 \mathrm{ng} \mathrm{ml}^{-1}$. Day 8 cultures were rinsed with $1 \times$ PBS and fixed for $5 \mathrm{~min}$ in ice-cold methanol-acetone $(50: 50)^{47}$. Following $6 \times 5$ min washes in $1 \times \mathrm{PBS}$, cultures were stained with rat anti-mouse PECAM-1 (BD Biosciences 553370) at $1: 1,000$ and then goat anti-rat IgG Alexa Fluor 488 (Life Technologies A-11006) at 1:2,000 overnight at $4{ }^{\circ} \mathrm{C}$ in Staining Solution (Jackson ImmunoResearch $005-000-121$ ), 5\% goat serum, 1\% BSA and 0.3\% TritonX-100 in DPBS (Fisher MT-21-031-CV).

HUVEC (Lonza C2519A) were maintained as per the manufacturer's recommendations and were used in experiments from passages 2 to 5 . All experiments were independently replicated using two distinct lots of HUVEC. HUVEC were certified mycoplasma-free by the UNC Tissue Culture Facility. Normal Human Lung Fibroblasts (NHLF; Lonza CC-2512) were maintained as per the manufacturer's recommendations. NHLF were certified mycoplasma-free by the UNC Tissue Culture Facility.

To induce Notch signalling, HUVEC were grown on recombinant protein $G$ (Life Technologies 10-1201)-immobilized $10 \mu \mathrm{g} \mathrm{ml}^{-1} \mathrm{Fc}-\mathrm{Dll} 4$ (Adipogen AG-40A-0077-C050) ${ }^{48}$ (or $10 \mu \mathrm{g} \mathrm{ml}^{-1} \mathrm{Fc}-\mathrm{IgG}$ control (Life Technologies 
10374-H02H-50)) for $24 \mathrm{~h}$, with BMP6 treatments and immunostaining performed as described above. For doxycycline induction, stably infected HUVEC FLAG-NICD cells or controls (see below) were treated for $24 \mathrm{~h}$ with $1-2 \mathrm{ng} \mathrm{ml}^{-1}$ doxycycline (Sigma-Aldrich D9891-1G) in growth media, then incubated with BMP6 $\left(200 \mathrm{ng} \mathrm{ml}^{-1}\right)$ for $2 \mathrm{~h}$, fixed in $4 \%$ paraformaldehyde (PFA) for $15 \mathrm{~min}$, rinsed three times with PBS, permeabilized with $0.5 \%$ Triton-X for $20 \mathrm{~min}$ and then blocked overnight with $1 \% \mathrm{BSA} / 0.3 \%$ Triton-X/5\% goat serum in PBS.

\section{Sprouting angiogenesis assay. The $3 \mathrm{D}$ sprouting angiogenesis assay was per-} formed as previously described ${ }^{21}$. Briefly, $1-2 \times 10^{6}$ HUVEC were mixed with $100 \mu \mathrm{l}$ of a $60,000 \mathrm{ml}^{-1}$ suspension of cytodex beads (GE Healthcare 17-0485-01) in $5 \mathrm{ml}$ HUVEC growth media (EBM-2 + EGM-2 BulletKit, Lonza CC-3156 and CC-3162, respectively), maintained in suspension for $4 \mathrm{~h}$ by mixing every $15 \mathrm{~min}$, then placed in $60 \mathrm{~mm}$ dishes at $37^{\circ} \mathrm{C}$ overnight. Beads were recovered from the plate by gentle rinsing and washed $3 \times$ in HUVEC growth media, then resuspended in $10 \mathrm{ml}^{\circ} 2 \mathrm{mg} \mathrm{ml}^{-1}$ fibrinogen $/ 0.15 \mathrm{U} \mathrm{ml}^{-1}$ aprotinin. Five microlitres of a $50 \mathrm{U} \mathrm{ml}^{-1}$ thrombin solution was added to each well of a 24-well plate, followed by gently mixing in $500 \mu \mathrm{l}$ of HUVEC-coated beads in fibrinogen/ aprotinin per well. After $5 \mathrm{~min}$ atRT, the plate was transferred to $37^{\circ} \mathrm{C}$ for $30 \mathrm{~min}$ for polymerization, then $1 \mathrm{ml}$ of 20,000 cells $\mathrm{ml}^{-1}$ NHLF was added to each well. Media was refreshed every 2 days until fixation. Recombinant human BMP2 or BMP6 (R\&D Systems \#355-BM-010 or \#507-BP-020, respectively) were added at d2-6 at $200 \mathrm{ng} \mathrm{ml}^{-1}$. Day 7 cultures were fixed in 2\% PFA (Electron Microscopy Supplies \#15713) in DPBS (Fisher \#MT-21-031-CV) for $30 \mathrm{~m}$, then stained with Alexa Fluor 488 Phalloidin or Alexa Fluor 594 Phalloidin (Life Technologies \#A12379 or \#A12381, respectively) overnight at $4{ }^{\circ} \mathrm{C}$ at 1:100 in Staining Solution (5\% Goat Serum (Jackson ImmunoResearch \#005-000-121), 1\% BSA, and 0.3\% TritonX-100 in DPBS). Nuclei were counterstained with DRAQ7 (Abcam \#ab109202) for $5 \mathrm{~min}$ at 1:1,000 in DPBS.

Immunofluorescence. For pSMAD1/5 immunofluorescence, HUVEC were grown for $24 \mathrm{~h}$ on $0.1 \%$ gelatin (Sigma-Aldrich \#G9136-10MG) coated $12 \mathrm{~mm}$ diameter \#1.5 glass coverslips (Fisher \#12-545-81). HUVEC were pretreated for $4 \mathrm{~h}$ in OptiMEM (Life Technologies \#31985-070) + 0.1\% normal bovine calf serum (NBCS), then treated for $90 \mathrm{~min}$ with BMP6 at the doses described in each figure. Cells were fixed for $10 \mathrm{~min}$ in $4 \%$ PFA/DPBS, followed by permeabilization in $0.5 \%$ Triton-X 100/DPBS. Cells were blocked in staining solution for $1 \mathrm{~h}$, followed by overnight incubation at $4{ }^{\circ} \mathrm{C}$ with Rabbit anti-pSMAD1/5 (R\&D) at 1:1,000 in staining solution (see Supplementary Table 1 for antibodies and concentrations). Goat anti-Rabbit Alexa Fluor 488 secondary antibody was added at 1:1,000 in staining solution for $2 \mathrm{~h}$ at room temperature (RT). For FLAG experiments, HUVEC were incubated with 1:1,000 $\alpha$-pSMAD $1 / 5$ antibody (R\&D) and 1:5,000 $\alpha$-FLAG antibody (Sigma) overnight, then washed and incubated with 1:500 Alexa Fluor 594 to detect pSMAD 1/5 and with 1:5,000 Alexa Fluor 488 to detect FLAG. DRAQ7 $(1: 1,000)$ was used to visualize the nucleus. Coverslips were mounted onto slides in ProLong Diamond Antifade Mountant (Life Technologies P36961).

For SMAD6 staining, HUVEC were incubated in 1:100 $\alpha$-SMAD6 (Abcam) and 1:5,000 $\alpha$-FLAG antibody (Sigma) overnight, and then with 1:500 Alexa Fluor 594 to detect SMAD6 and with 1:5,000 Alexa Fluor 488 to detect the FLAG antibody. Alexa Fluor 647-conjugated Phalloidin was used to visualize cell boundaries.

Plasmids. To generate a lentiviral vector expressing human SMAD6-tdTomato fusion protein under the control of a doxycycline-inducible promoter, the full-length human SMAD6 coding sequence was amplified from pOTB7 hSMAD6 (GE Dharmacon MHS6278-202829590) using PrimeSTAR MAX polymerase (Clontech $\mathrm{R} 045 \mathrm{~A}$ ) and the following primers:

Forward: 5'-ATTCACAGATCTGCCACCATGTTCAGGTCCAAACGCTCG-3', containing $6 \mathrm{nt}$ overhang, BglII restriction site and Kozak consensus sequence,

Reverse: 5' -ATTCACGGTACCCTTCTGGGGTTGTTGAGGAGGATCTC-3', containing $6 \mathrm{nt}$ overhang, KpnI restriction site and $1 \mathrm{nt}$ deletion from stop codon for in-frame read-through to carboxy-terminal tdTomato tag.

PCR product was purified using NucleoSpin kit (Clontech 740609) and double-digested for $10 \mathrm{~min}$ at $37^{\circ}$ with BglII and KpnI (Life Technologies FD0084 and FD0524, respectively). ptdTomato-N1 empty vector (Clontech 632532) was double-digested in parallel using the same enzymes and de-phosphorylated for 5 min at $37^{\circ}$ using FastAP Thermosensitive Alkaline Phosphatase (Life Technologies EF0654). Products were ligated at a 3:1 (Insert:Vector) molar ratio for 10 min at RT using T4 Rapid Ligation Kit (Life Technologies K1422), followed by transformation, selection, plasmid purification and sequencing, to verify the fidelity of the insert.

The full-length hSMAD6-tdTomato chimera was PCR-amplified and cloned into gateway-compatible pME-MCS ${ }^{49}$ from the newly generated template using a similar strategy.

Forward primer: $5^{\prime}$-ATTCACAAGCTTGCCACCATGTTCAGGTCCAAACGC TCG-3', containing $6 \mathrm{nt}$ overhang, HindIII (Life Technologies FD0504) restriction site and Kozak consensus sequence,

Reverse primer: 5'-ATTCACTCTAGACTACTTGTACAGCTCGTCCAT-3', containing 6 nt overhang and XbaI (Life Technologies FD0684) restriction site.
An LR reaction was performed using Clonase II Plus (Life Technologies 12538-120), pME-hSAMD6-tdTomato and pLIX-402 EV (a gift from David Root (Addgene plasmid 41394)) to generate pLIX-402 hSMAD6-tdTomato.

pLIX-402 $3 \times$-FLAG-mN1ICD was generated from pCMV-7 $3 \times$-FLAG mN1ICD $^{50}$ (a gift from Raphael Kopan, Addgene plasmid 20183) by double digestion and ligation into pME-MCS as described above, using FastDigest EcoRI and BamHI (Life Technologies FD0274 and FD0054, respectively), followed by an LR reaction.

Zebrafish transgenesis was performed using the Tol2 system ${ }^{49}$. To generate the pTol2 fli1:smad6b-GFP;cmlc2:GFP targeting vector, full-length smad $6 b$ was amplified from a $24 \mathrm{hpf}$ embryo complementary DNA library using PrimeSTAR Max and cloned into pCS2 FLAG EV (a gift from Peter Klein, Addgene plasmid 16331) as described above, using the following primers:

Forward: 5'-ATTCACGGATCCGCCACCATGTTCAGGACGAAACGC

TCA-3', containing a $6 \mathrm{nt}$ overhang, BamHI site and Kozak consensus sequence,

Reverse: $5^{\prime}$-ATTCACATCGATATCTGTGGTTGTTGAGGAGG-3', containing a 6 nt overhang, ClaI (Life Technologies FD0143) site and 2 nt deletion from the stop codon to maintain frame with C-terminal tags.

C-terminal FLAG tag was replaced with GFP by amplifying GFP from peGFP-N1 EV (Clontech 6085-1), digested and ligated as described above, using the following primers:

Forward: 5'-ATTCACATCGATTGGTGAGCAAGGGCGAGGAGC-3', containing a 6 nt overhang, ClaI site and a 1 nt deletion from the start codon,

Reverse: 5'-ATTCACAGGCCTTTACTTGTACAGCTCGTCCAT-3',

containing a $6 \mathrm{nt}$ overhang and StuI (NEB R0187S) site.

Smad6b-GFP was then ligated into pME-MCS using a BamHI and NotI (Life Technologies FD0594) double digest as described above. A four-way LR reaction using p5E-flilep (a gift from Nathan Lawson, Addgene plasmid 31160), pME-smad6b-GFP, p3E-polyA ${ }^{49}$ and pDEST Tol2 CG2 (ref. 49) produced the final targeting vector.

To generate the pTol2-flilep:Cas9-EnR;cmlc2:GFP targeting vector, $d$ Cas 9 was amplified from pdCas9 (ref. 41; a gift from Stanley Qi, Addgene plasmid 44246) and ligated into pCS2-EnR $R^{43}$ (a gift from Ramesh Shivdasani, Addgene plasmid 11028) using the following primers:

Forward: 5'-GGATCCGCCACCATGGACAAGAAGTATTCTATC-3', containing a 6 nt overhang, BamHI site and Kozak consensus sequence, Reverse: $5^{\prime}$-ATTCACATCGATTACCAATGCCGTCTACCTT TCTCTTCTTTTTTGGATCTACCTTTCTCTTCTTTTTTGGATCTACCTT-3', containing a $6 \mathrm{nt}$ overhang, ClaI site and deletion of the stop codon.

$d$ Cas $9-E n R$ was subcloned into pME-MCS using BamHI/XbaI double digests and four-way LR reaction performed with the same $5 \mathrm{E}, 3 \mathrm{E}$ and DEST vectors described above.

Lentivirus production and stably infected HUVEC. Lenti-X 293T cells (Clontech 632180) were maintained in DMEM Hi Glucose (Life Technologies 11995-065) $+10 \%$ normal bovine calf serum (NBCS) according to the manufacturer's instructions. For lentiviral production, cells were plated onto $10 \mathrm{~cm}$ dishes at $50,000 \mathrm{~cm}^{-2}$. The following morning, cells were co-transfected with $20 \mu \mathrm{g}$ pLIX 402 vectors (described above) and $6 \mu \mathrm{g}$ pMD2.G (a gift from Didier Trono, Addgene plasmid 12259), $5 \mu \mathrm{g}$ pRSV-REV ${ }^{51}$ (a gift from Didier Trono, Addgene plasmid 12253) and $10 \mu \mathrm{g}$ pMDLg/pRRE ${ }^{51}$ (a gift from Didier Trono, Addgene plasmid 12251) using Lipofectamine 2000 (Life Technologies 11668019) according to the manufacturer's protocol. Transfection medium was aspirated $8 \mathrm{~h}$ later and $6 \mathrm{ml}$ fresh medium added. Forty-eight hours later, medium was harvested by centrifugation, filtered through a sterile $0.22 \mu \mathrm{m}, 30 \mathrm{~mm}$ PES filter (Fisher Scientific 50-202-062), mixed 1:1 with HUVEC growth media (EBM-2 + EGM-2 BulletKit, Lonza CC-3156 and CC-3162, respectively) and added to $80 \%$ confluent p2 HUVEC. The next day, medium was aspirated and fresh medium added. After $72 \mathrm{~h}$, infected HUVEC were selected using $1 \mathrm{ng} \mathrm{ml}^{-1}$ puromycin (Sigma-Aldrich P9620-10 ml) in HUVEC growth medium, changed daily, for 2 weeks. Surviving HUVEC were then trypsinized, re-plated under $0.5 \mathrm{ng} \mathrm{ml}^{-1}$ puromycin selection and expanded.

siRNA transfections. siRNAs used in this study are listed in Supplementary Table 2. HUVEC were plated at $20,000 \mathrm{~cm}^{-2}$ onto $6 \mathrm{~cm}$ dishes. The following day, $\sim 80 \%$ confluent HUVEC were transfected with $10 \mathrm{nM}$ siRNA using Lipofectamine RNAiMax (Life Technologies 13778150) according to the manufacturer's protocol. The following day, HUVEC were trypsinized, coated onto Cytodex beads for 3D experiments or plated onto $12 \mathrm{~mm}$ coverslips for two-dimensional (2D) experiments and re-transfected with siRNA to ensure maximal knockdown persisted throughout the duration of the experiments. Residual cells were analysed by western blotting, to verify knockdown efficacy.

Western blotting. HUVEC whole-cell lysates were prepared in RIPA buffer supplemented with protease/phosphatase inhibitor cocktail (Cell Signaling 5872S). Ten micrograms of whole-cell lysates were separated on $10 \%$ TGX Stain-Free FastCast SDS-PAGE gels (Bio-Rad 161-0183), followed by 2 min ultraviolet activation of TGX stain for total protein quantification. Proteins were transferred to polyvinylidene difluoride membranes (Bio-Rad 162-0177), blots were imaged 
under ultraviolet for total protein, blocked for $2 \mathrm{~h}$ in $5 \%$ non-fat milk (NFM) in PBS $+0.1 \%$ Tween 20 (PBST), then incubated overnight at $4{ }^{\circ} \mathrm{C}$ with primary antibody in 1\% NFM in PBST (see Supplementary Table 1 for primary and secondary antibodies and concentrations). Horseradish peroxidase-conjugated secondary antibodies (Life Technologies goat anti-rabbit G21234 or rabbit anti-mouse 816720 ) were added for $2 \mathrm{~h}$ at RT in $1 \%$ NFM in PBST. Clarity ECL (Bio-Rad 170-5060) was used for detection. Films were digitized using an AlphaImager (ProteinSimple) gel scanning station and band densities calculated in FIJI $^{52}$, following established guidelines ${ }^{53}$.

Quantitative RT-PCR. Primers used in this study are listed in Supplementary Table 3. mRNA was collected from experimental samples using Trizol reagent (Life Technologies 15596026). cDNA was generated from $1 \mu \mathrm{g}$ mRNA using iScript reverse transcription kit (Bio-Rad 170-8891) and diluted 1:3 in water. qRT-PCR was performed using iTaq Universal SYBR Green SuperMix (Bio-Rad 172-5121) on an ABI 7900HT Fast Real-Time PCR System (Life Technologies 4329001). Threefold serial dilutions of pooled cDNA were used to generate standard curves for each amplicon and data were analysed via the Pfaffl method ${ }^{54}$.

RNA was isolated from FACS-enriched zebrafish cells using RNA-easy Micro Plus kit (Qiagen) and reverse transcribed with Invitrogen's Superscript III First-Strand Synthesis Supermix (Invitrogen) according to the manufacturer's instructions. For qRT-PCR, NCBI's Primer-BLAST was used to design exon-spanning, gene-specific SybrGreen primers (see Supplementary Table 3). All primers were validated by high-resolution melt analysis, size confirmation and no-template controls. SybrGreen real-time PCR was performed in triplicate on the Viia7 real-time PCR system (Invitrogen). For quantification, the $\Delta \Delta \mathrm{CT}$ method was used where raw CT values were normalized to elongation factor alpha (eefla1l1) and paired sorting control, then calculated fold change as $2^{(-\Delta \Delta C T)}$. Statistical significance was determined by one-sample $T$-test comparing with a reference value of onefold change.

Zebrafish. Zebrafish (Danio rerio) embryos were maintained as previously described $^{9}$. The following transgenic lines were used: $\operatorname{Tg}(k d r l: G F P)^{s 843}$ (ref. 55),

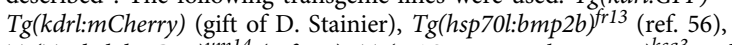
Tg(Tp1bglob:eGFP) um14 (ref. 57), Tg(UAS:myc-Notchla-intra) ${ }^{k c a 3}$ and $\mathrm{Tg}$ (hsp70l:Gal4) ${ }^{1.5 k c a 4}$ (ref. 58), and $\mathrm{Tg}$ (flilep:dCas9-EnR;cmlc2:GFP) (this study).

For all microinjections, one-cell-stage embryos were dechorionated using $20 \mathrm{mg} \mathrm{ml}^{-1}$ (W/V in $\mathrm{ddH}_{2} \mathrm{O}$ ) pronase (Sigma P6911), then rinsed repeatedly using a total of $11 \mathrm{H}$ Buffer $\left(60 \mathrm{mM} \mathrm{NaCl}, 2.3 \mathrm{mM} \mathrm{NaHCO}_{3}, 1.1 \mathrm{mM} \mathrm{CaCl}_{2}\right.$ and $0.67 \mathrm{mM} \mathrm{KCl}$ ).

For Notch inhibition, embryos were treated with DAPT at $100 \mu \mathrm{M}$, sonicated in $1 \%$ dimethyl sulfoxide/1 $\times 1$-phenyl 2-thiourea $(\mathrm{PTU}) / \mathrm{E} 3$, or in $1 \%$ dimethyl sulfoxide/1 $\times$ 1-phenyl 2-thiourea (PTU)/E3 control, from $10 \mathrm{hpf}$ until harvest. For Notch or BMP activation, embryos were heat-shocked at $26 \mathrm{hpf}$ for $30 \mathrm{~min}$ at $40^{\circ} \mathrm{C}$ or kept as unheat-shocked controls. For transgenesis, capped mRNA was generated from pCS2 FA Transposase ${ }^{49}$ using the T7 mMessage mMachine in vitro synthesis kit (Life Technologies AM1344). Five picograms each of transposase mRNA and Tol2 vector were injected into the cell of dechorionated one-cell-stage zebrafish embryos from $A B$ (flilep:dCas9-EnR;cmlc2:GFP) or Tg(kdrl:mCherry

(fli1ep:smad6b-GFP;cmlc2:GFP), or flilep:GFP;cmlc2:GFP) in-crosses. Embryos were screened for cardiac GFP expression (transgenesis) and raised until harvest as F0 mosaics (flilep:smad6b-GFP or flilep:GFP) or raised to adulthood and screened for germline transmission (flilep:dCas9-EnR;cmlc2:GFP).

For global CRISPRi, WT embryos from AB parents were injected at the one-cell stage with $450 \mathrm{pg}$ dCas9-EnR mRNA alone or in conjunction with $200 \mathrm{pg}$ gene-targeting sgRNA. Embryos were analysed at $24 \mathrm{hpf}$ for gross dorsal-ventral patterning defects consistent with early BMP manipulations ${ }^{38}$. For CRISPRi, $>50$ embryos from $T g(h s p: b m p 2 b) ; T g(k d r l: G F P) \times T g(f l i l e p: d C a s 9-E n R ; c m l c 2: G F P)$ crosses were injected using a PicoSpritzer III positive pressure micro-injection apparatus at the one-cell-stage with $200 \mathrm{pg}$ (in $1 \mathrm{nl}$ ) of scrambled (scram) or smad $6 b$-targeting sgRNAs. Embryos were raised at $28^{\circ} \mathrm{C}$ in $1 \times \mathrm{E} 3$ and heatshocked to overexpress Bmp $2 \mathrm{~b}$ at $26 \mathrm{hpf}$ as described above. Embryos were fixed and imaged on an FV1200 confocal microscope at $44 \mathrm{hpf}$. sgRNA sequences used are listed in Supplementary Table 3.

For all zebrafish experiments, embryos were collected and sorted into treatments blindly with respect to genotype, to ensure appropriate randomization. For all experiments, any embryos that failed to gastrulate or exhibited severe developmental patterning defects were excluded from analysis. All animals are maintained in an institutional animal care and use committee (IACUC)-approved satellite facility according to public health service (PHS) policy on Humane Care and Use of Laboratory Animals.

FACS sorting. Staged fish embryos were obtained by breeding hemizygous $\operatorname{Tg}(T p 1: E G F P)$; $\operatorname{Tg}($ myl7:dsRed) adult zebrafish with homozygous $\operatorname{Tg}(f l i 1: t d T o m a t o$; cmlc2:GFP) fish by group spawning methods. Non-transgenic embryos (for use in setting FACS gates) were obtained by inbreeding WT (Tubingen) fish. Embryos from individual spawning groups were pooled into single clutch biological replicates. At $26 \mathrm{hpf}$, embryos were manually sorted for Tp1:EGFP expression. At $28-32 \mathrm{hpf}$, embryos were prepared for FACS sorting as previously described ${ }^{19}$.
Briefly, embryos were manually dechorinated, yolk removed, then dissociated into single-cell suspension using a combination of TrypLE (Gibco) and FACSMax (Gentlantis). Cells were re-suspended in ice-cold L-15 (Gibco) supplemented with $5 \%$ heat-inactivated FBS (Hyclone), passed through 40 and $35 \mu \mathrm{m}$ filters, then counterstained with Live/Dead fixable near-IR stain (Molecular Probes). A SH800 cell sorter (Sony) was used to sort live, tdTomato + cells by Tp1:EGFP expression level and harvested directly into RTL lysis buffer, then stored at -20 for later RNA purification.

ChIP assay. HUVEC were plated on $100 \mathrm{~mm}$ culture dishes and grown for $24 \mathrm{~h}$ and native protein-DNA complexes were cross-linked by treatment with $1 \%$ formaldehyde for $15 \mathrm{~min}$. Simple ChIP Plus Enzymatic Chromatin IP kit (Cell Signaling 9005) was used per the manufacturer's protocol. Briefly, equal aliquots of isolated chromatin were subjected to immunoprecipitation with anti-RBPJ antibodies (Cell Signaling 5313s, Abcam ab25949) or rabbit IgG control at $2 \mu \mathrm{g}$ per $500 \mu \mathrm{l}$. PCR reactions of immunoprecipitated DNA were performed to validate RBPJ binding on the Smad6 promoter. PCR primers used:

2: FWD: $5^{\prime}$-ATTAGCCGGGCATAGTGGTGCAT-3'

REV: $5^{\prime}$-TGCACTCAAGTGATTCTCGTGCCT-3'

3: FWD: 5'-AGGCGGATCACTTCAGGCAGGTCA- $3^{\prime}$

REV: 5'-GCTCTGCACTCAAGTGATTCTCGT- $3^{\prime}$.

PCR products were separated by gel electrophoresis and visualized by SYBRsafe (Invitrogen).

Imaging and quantification. All images were acquired on an Olympus FluoView FV1200 confocal microscope, equipped with Green, Red and Far-Red lasers and detectors. Olympus OIB file formats were imported into FIJI using Bio-Formats Importer ${ }^{20}$ for analysis and quantification, as described below.

Branch frequency was measured by skeletonizing ES-cell-derived vessels or HUVEC-derived 3D sprouting angiogenesis assay vessels and calculating branches per $\mathrm{mm}$ using Image $\mathrm{J}(\mathrm{NIH})$. For branching parameters, the sprout length was measured from the base of the spout to the most distal end. The maximum branch angle was calculated at sprout junctions, excluding branches that fused with other sprouts distal to the junction. The percentage of tip cells was quantified in all multinucleated sprouts. Tip cells were defined as the cell nucleus located most distal in a sprout. In zebrafish, the percentage of somites containing angiogenic sprouts was calculated. The first 12 segments from the end of yolk extension were analysed as described ${ }^{9}$.

pSMAD1/5 fluorescence intensities were determined on a single-cell basis in 2D using FIJI as follows: HUVEC were stained for pSMAD1/5 (also FLAG if relevant) and incubated with DRAQ7 as described in detail above. The brightest slice in the DRAQ7 (nuclear) channel from confocal $z$-stack images was threshold adjusted into a binary image (black nuclei, white everywhere else). Next, using the 'Analysis' $\rightarrow$ 'Set Measurements' menu, analysis was re-directed from the binary nuclear image to the pSMAD1/5 channel and 'Analyze' $\rightarrow$ 'Analyze Particles' function was used with area cutoffs of $50-500 \mu \mathrm{m}^{2}$ to return mean grey values per nucleus.

pSMAD1/5 fluorescence intensities were determined on a single-cell basis in 3D using FIJI as follows: pSMAD1/5 and DRAQ7 (nuclear) channels were independently compressed along the $z$ axis by summing slices. DRAQ7 channel was threshold adjusted as described above and binary image used to re-direct measurements to the pSMAD1/5 channel as described above. The geodesic distance from the tip nucleus was measured along the length of the vessel from the most distal portion of the tip nucleus. For tip-cell distance:pSMAD1/5 fluorescence correlations, both distance and fluorescence were determined relative to the tip cell ( $0 \mu \mathrm{m}$ distance and $100 \%$ fluorescence).

SMAD6 fluorescence intensities were determined on a single-cell basis in $2 \mathrm{D}$ as follows: HUVEC were stained for SMAD6 and FLAG, and incubated with Phalloidin to visualize cell boundaries as described in detail above. SMAD6 fluorescence intensity was measured by finding the brightest slice in the phalloidin channel, outlining individual cells and calculating integrated density within the SMAD6 channel with subtraction of background.

Statistics. All statistical analyses were performed using Prism v6.05 (www.graphpad.com), with an $\alpha$ of 0.05 . Data were tested for normal distribution using the D'Agostino and Pearson omnibus normality test within Prism. For two-sample data sets with equal variances (control -v- a single experimental condition) unpaired, two-tailed Student's $t$-test was used as reported in figure legends. For data sets with greater than two conditions and equal variances, one-way analysis of variance with Tukey's post-hoc test was used as reported in figure legends. For data sets with greater than two conditions and unequal variances, Kruskal-Wallis with Dunn's post-hoc test was used as reported in figure legends. No a priori sample-size power analyses were performed.

Data availability. Data supporting the findings of this work are available within the article and its Supplementary Information files, and from the corresponding author on reasonable request. 


\section{References}

1. Adams, R. H. \& Alitalo, K. Molecular regulation of angiogenesis and lymphangiogenesis. Nat. Rev. Mol. Cell Biol. 8, 464-478 (2007).

2. Lowery, J. W. \& de Caestecker, M. P. BMP signaling in vascular development and disease. Cytokine Growth Factor Rev. 21, 287-298 (2010).

3. Eilken, H. M. \& Adams, R. H. Dynamics of endothelial cell behavior in sprouting angiogenesis. Curr. Opin. Cell Biol. 22, 617-625 (2010).

4. Geudens, I. \& Gerhardt, H. Coordinating cell behaviour during blood vessel formation. Development 138, 4569-4583 (2011)

5. Chappell, J. C., Wiley, D. M. \& Bautch, V. L. Regulation of blood vessel sprouting. Semin. Cell Dev. Biol. 22, 1005-1011 (2011).

6. Bentley, K. et al. The role of differential VE-cadherin dynamics in cell rearrangement during angiogenesis. Nat. Cell Biol. 16, 309-321 (2014)

7. Phng, L. K. \& Gerhardt, H. Angiogenesis: a team effort coordinated by Notch. Dev. Cell 16, 196-208 (2009).

8. Moya, I. M. et al. Stalk cell phenotype depends on integration of Notch and Smad1/5 signaling cascades. Dev. Cell 22, 501-514 (2012)

9. Wiley, D. M. et al. Distinct signalling pathways regulate sprouting angiogenesis from the dorsal aorta and the axial vein. Nat. Cell Biol. 13, 686-692 (2011).

10. van Meeteren, L. A. et al. Anti-human activin receptor-like kinase 1 (ALK1) antibody attenuates bone morphogenetic protein 9 (BMP9)-induced ALK1 signaling and interferes with endothelial cell sprouting. J. Biol. Chem. 287, 18551-18561 (2012).

11. Suzuki, Y. et al. BMP-9 induces proliferation of multiple types of endothelial cells in vitro and in vivo. J. Cell Sci. 123, 1684-1692 (2010).

12. Ramoshebi, L. N. \& Ripamonti, U. Osteogenic protein-1, a bone morphogenetic protein, induces angiogenesis in the chick chorioallantoic membrane and synergizes with basic fibroblast growth factor and transforming growth factorbetal. Anat. Rec. 259, 97-107 (2000).

13. Larrivee, B. et al. ALK1 signaling inhibits angiogenesis by cooperating with the Notch pathway. Dev. Cell 22, 489-500 (2012)

14. Zhou, Q. et al. ERK signaling is a central regulator for BMP-4 dependent capillary sprouting. Cardiovasc. Res. 76, 390-399 (2007).

15. Bragdon, B. et al. Bone morphogenetic proteins: a critical review. Cell Signal. 23, 609-620 (2011).

16. Derynck, R. \& Zhang, Y. E. Smad-dependent and Smad-independent pathways in TGF-beta family signalling. Nature 425, 577-584 (2003).

17. Hata, A., Lagna, G., Massague, J. \& Hemmati-Brivanlou, A. Smad6 inhibits BMP/Smad1 signaling by specifically competing with the Smad4 tumor suppressor. Genes Dev. 12, 186-197 (1998).

18. Imamura, T. et al. Smad6 inhibits signalling by the TGF-beta superfamily. Nature 389, 622-626 (1997).

19. Yang, X. et al. Angiogenesis defects and mesenchymal apoptosis in mice lacking SMAD5. Development 126, 1571-1580 (1999).

20. Kim, J. D. et al. Context-dependent proangiogenic function of bone morphogenetic protein signaling is mediated by disabled homolog 2. Dev. Cell 23, 441-448 (2012)

21. Nakatsu, M. N., Davis, J. \& Hughes, C. C. Optimized fibrin gel bead assay for the study of angiogenesis. J. Vis. Exp. 186 (2007).

22. Chappell, J. C., Taylor, S. M., Ferrara, N. \& Bautch, V. L. Local guidance of emerging vessel sprouts requires soluble Flt-1. Dev. Cell 17, 377-386 (2009).

23. Chappell, J. C., Mouillesseaux, K. P. \& Bautch, V. L. Flt-1 (vascular endothelial growth factor receptor-1) is essential for the vascular endothelial growth factor-Notch feedback loop during angiogenesis. Arterioscler. Thromb. Vasc. Biol. 33, 1952-1959 (2013).

24. David, L., Mallet, C., Mazerbourg, S., Feige, J. J. \& Bailly, S. Identification of BMP9 and BMP10 as functional activators of the orphan activin receptor-like kinase 1 (ALK1) in endothelial cells. Blood 109, 1953-1961 (2007).

25. David, L. et al. Bone morphogenetic protein- 9 is a circulating vascular quiescence factor. Circ. Res. 102, 914-922 (2008).

26. Hellstrom, M. et al. Dll4 signalling through Notch1 regulates formation of tip cells during angiogenesis. Nature 445, 776-780 (2007)

27. Leslie, J. D. et al. Endothelial signalling by the Notch ligand Delta-like 4 restricts angiogenesis. Development 134, 839-844 (2007).

28. Suchting, S. et al. The Notch ligand Delta-like 4 negatively regulates endothelial tip cell formation and vessel branching. Proc. Natl Acad. Sci. USA 104, 3225-3230 (2007)

29. Jakobsson, L. et al. Endothelial cells dynamically compete for the tip cell position during angiogenic sprouting. Nat. Cell Biol. 12, 943-953 (2010).

30. Itoh, F. et al. Synergy and antagonism between Notch and BMP receptor signaling pathways in endothelial cells. EMBO J. 23, 541-551 (2004).

31. Maddaluno, L. et al. EndMT contributes to the onset and progression of cerebral cavernous malformations. Nature 498, 492-496 (2013).

32. Wythe, J. D. et al. ETS factors regulate Vegf-dependent arterial specification. Dev. Cell 26, 45-58 (2013).

33. Quillien, A. et al. Distinct Notch signaling outputs pattern the developing arterial system. Development 141, 1544-1552 (2014).
34. Harrington, L. S. et al. Regulation of multiple angiogenic pathways by Dll4 and Notch in human umbilical vein endothelial cells. Microvasc. Res. 75, 144-154 (2008).

35. Funahashi, Y. et al. Notch regulates the angiogenic response via induction of VEGFR-1. J. Angiogenes Res. 2, 3 (2010).

36. Afrakhte, M. et al. Induction of inhibitory Smad6 and Smad7 mRNA by TGF-beta family members. Biochem. Biophys. Res. Commun. 249, 505-511 (1998).

37. Ishida, W. et al. Smad6 is a Smad1/5-induced smad inhibitor. Characterization of bone morphogenetic protein-responsive element in the mouse Smad6 promoter. J. Biol. Chem. 275, 6075-6079 (2000).

38. Kishimoto, Y., Lee, K. H., Zon, L., Hammerschmidt, M. \& Schulte-Merker, S. The molecular nature of zebrafish swirl: BMP2 function is essential during early dorsoventral patterning. Development 124, 4457-4466 (1997).

39. Gilbert, L. A. et al. CRISPR-mediated modular RNA-guided regulation of transcription in eukaryotes. Cell 154, 442-451 (2013).

40. Larson, M. H. et al. CRISPR interference (CRISPRi) for sequence-specific control of gene expression. Nat. Protoc. 8, 2180-2196 (2013).

41. Qi, L. S. et al. Repurposing CRISPR as an RNA-guided platform for sequence-specific control of gene expression. Cell 152, 1173-1183 (2013).

42. Rossi, A. et al. Genetic compensation induced by deleterious mutations but not gene knockdowns. Nature 524, 230-233 (2015).

43. Shoichet, S. A., Malik, T. H., Rothman, J. H. \& Shivdasani, R. A. Action of the Caenorhabditis elegans GATA factor END-1 in Xenopus suggests that similar mechanisms initiate endoderm development in ecdysozoa and vertebrates. Proc. Natl Acad. Sci. USA 97, 4076-4081 (2000).

44. Siekmann, A. F. \& Lawson, N. D. Notch signalling limits angiogenic cell behaviour in developing zebrafish arteries. Nature 445, 781-784 (2007).

45. Aspalter, I. M. et al. Alk1 and Alk5 inhibition by Nrp1 controls vascular sprouting downstream of Notch. Nat. Commun. 6, 7264 (2015).

46. Kearney, J. B. \& Bautch, V. L. In vitro differentiation of mouse ES cells hematopoietic and vascular development. Methods Enzymol. 365, 83-98 (2003).

47. Bautch, V. L. et al. Characterization of the vasculogenic block in the absence of vascular endothelial growth factor-A. Blood 95, 1979-1987 (2000).

48. Varnum-Finney, B. et al. Immobilization of Notch ligand, Delta-1, is required for induction of notch signaling. J. Cell Sci. 113 Pt 23, 4313-4318 (2000).

49. Kwan, K. M. et al. The Tol2kit: a multisite gateway-based construction kit for Tol2 transposon transgenesis constructs. Dev. Dyn. 236, 3088-3099 (2007).

50. Ong, C. T. et al. Target selectivity of vertebrate notch proteins. Collaboration between discrete domains and CSL-binding site architecture determines activation probability. J. Biol. Chem. 281, 5106-5119 (2006).

51. Dull, T. et al. A third-generation lentivirus vector with a conditional packaging system. J. Virol. 72, 8463-8471 (1998).

52. Schindelin, J. et al. Fiji: an open-source platform for biological-image analysis Nat. Methods 9, 676-682 (2012).

53. Gassmann, M., Grenacher, B., Rohde, B. \& Vogel, J. Quantifying Western blots: pitfalls of densitometry. Electrophoresis 30, 1845-1855 (2009).

54. Pfaffl, M. W. A new mathematical model for relative quantification in real-time RT-PCR. Nucleic Acids Res. 29, e45 (2001).

55. Jin, S. W., Beisl, D., Mitchell, T., Chen, J. N. \& Stainier, D. Y. R. Cellular and molecular analyses of vascular tube and lumen formation in zebrafish. Development 132, 5199-5209 (2005).

56. Chocron, S., Verhoeven, M. C., Rentzsch, F., Hammerschmidt, M. \& Bakkers, J Zebrafish Bmp4 regulates left-right asymmetry at two distinct developmental time points. Dev. Biol. 305, 577-588 (2007).

57. Parsons, M. J. et al. Notch-responsive cells initiate the secondary transition in larval zebrafish pancreas. Mech. Dev. 126, 898-912 (2009).

58. Scheer, N. \& Campos-Ortega, J. A. Use of the Gal4-UAS technique for targeted gene expression in the zebrafish. Mech. Dev. 80, 153-158 (1999).

\section{Acknowledgements}

We thank Bautch lab members for constructive discussion and input, and Drs Andrew Dudley and Frank Conlon for comments on the manuscript. This work was supported by NIH grants HL43174 and HL116719 (V.L.B.); GIST Research Institute (GRI), Cell Logistics Research Center, National Research Foundation (NRF-2016R1A5A1007318) of Korea and NIH grants HL090960 and HL114820 to S.W.J.; the NRF of Korea (NRF-2013R1A1A1057591) to J.K.; an NIH NRSA (F32HL123264-02) to K.P.M and AHA Predoctoral Fellowship (10PRE3290010) to D.S.W. We also thank Michelle Ortwine and the UNC Zebrafish Aquaculture Core Facility for excellent care of adult fish and the use of microinjection facilities.

\section{Author contributions}

K.P.M. and D.S.W. made equal contributions to design, experimental execution and interpretation. K.P.M., D.S.W., S-W.J. and V.L.B. designed and interpreted the study. K.P.M., D.S.W., L.M.S., L.A.W., E.J.K., D.C.C., K.M.C., Y.P., J-D.K. and L.A.S. performed experiments. K.P.M., D.S.W., L.M.S., L.A.W., E.J.K., D.C.C., K.M.C., A.T.B., Y.P., J-D.K and L.A.S. collected and analysed data. J.K., J.L., S-W.J. and V.L.B. supervised and supported data acquisition. V.L.B. and K.M.P. wrote and edited the manuscript. D.S.W., S-W.J., L.M.S., L.A.W., D.C.C. and L.A.S. edited the manuscript. 


\section{Additional information}

Supplementary Information accompanies this paper at http://www.nature.com/ naturecommunications

Competing financial interests: The authors declare no competing financial interests.

Reprints and permission information is available online at http://npg.nature.com/ reprintsandpermissions/

How to cite this article: Mouillesseaux, K. P. et al. Notch regulates BMP responsiveness and lateral branching in vessel networks via SMAD6. Nat. Commun. 7, 13247 doi: 10.1038/ncomms13247 (2016).
Publisher's note: Springer Nature remains neutral with regard to jurisdictional claims in published maps and institutional affiliations.

(c) (i) This work is licensed under a Creative Commons Attribution 4.0 International License. The images or other third party material in this article are included in the article's Creative Commons license, unless indicated otherwise in the credit line; if the material is not included under the Creative Commons license, users will need to obtain permission from the license holder to reproduce the material. To view a copy of this license, visit http://creativecommons.org/licenses/by/4.0/

(C) The Author(s) 2016 